\title{
Uma iniciativa para atrair as estudantes do ensino médio para a Área de Tecnologia da Informação
}

\author{
Marina Macedo Nunes ${ }^{1}$, Larissa Ferreira Rodrigues ${ }^{1}$, Adriana Zanella \\ Martinhago $^{1}$, Liziane Santos Soares ${ }^{1}$, Rachel Carlos Duque Reis ${ }^{1}$ \\ ${ }^{1}$ Instituto de Ciências Exatas e Tecnológicas - Universidade Federal de Viçosa - Campus \\ Rio Paranaíba, Rio Paranaíba - MG - Brasil \\ \{marina.nunes, larissa.f.rodrigues, adriana.martinhago, \\ liziane.soares, rachel.reis\} dufv.br
}

\begin{abstract}
Several organizations around the world are focused on issues related to minority of women in Information Technology (IT) area. The development of this area is related to the development of science, technology and innovation of any nation or organization. However the IT area presents a lack of trained professionals. If we consider the participation of women in this sector, the lack is even greater. This work consists on the development of actions to attract high school women students to IT area. During 2014, this work held several meetings in a high school, including lectures, short courses and dynamics, introducing computing and programming fundamentals. These actions contributed to the student's education, making them more prepared to work in a society where science and innovation play a key role. Until now it was observed that work aroused the curiosity of women students about IT area and most of them indicated that would like to participate in new projects focused on computing area.
\end{abstract}

Resumo. Muitas organizações ao redor do mundo estão voltadas para o tema da minoria das mulheres na área de Tecnologia da Informação - TI. O desenvolvimento dessa área está intimamente ligado ao desenvolvimento da ciência, tecnologia e inovação de qualquer nação ou organização. Contudo a área de TI apresenta uma carência de profissionais capacitados. Se considerarmos a participação feminina neste setor, a carência é ainda mais expressiva. Este trabalho consiste no desenvolvimento de ações visando atrair alunas do ensino médio para a área de Tecnologia da Informação. Durante o ano de 2014, este trabalho realizou diversos encontros em uma escola do ensino médio, incluindo palestras, minicursos e dinâmicas e introduzindo fundamentos da computação e da programação. Essas ações contribuíram para a formação complementar dos estudantes, tornando-os mais preparados para atuar em uma sociedade onde ciência e inovação tem um papel fundamental. Até o momento foi possivel observar que trabalho despertou a curiosidade das alunas sobre a área de TI e a maioria delas sinalizou que gostaria de participar de novos projetos focados na área de computação. 


\section{Introdução}

Muitas organizações ao redor do mundo estão voltadas para o tema da minoria das mulheres na área de Tecnologia da Informação - TI (Computação, Sistemas de Informação e áreas afins). Atualmente, o desenvolvimento de qualquer nação está pautado na necessidade de recursos humanos que apresentem habilidades e treinamento na área de TI. O MCT-Ministério de Ciência, Tecnologia e Inovação, através do TI Maior $^{1}$ (Programa Estratégico de Software e Serviços de TI) e da ENCTI (Estratégia Nacional de Ciência, Tecnologia e Inovação) reforçam ainda mais a importância da área de TI para o desenvolvimento da ciência, tecnologia e inovação no país.

De forma geral, observa-se uma carência de profissionais nesta área tanto em nível nacional como internacional [Venceslau 2013]. Se considerarmos a participação feminina neste setor, essa carência é ainda mais expressiva. O desinteresse das mulheres por essa área é percebido desde o momento da escolha do curso de graduação. O número de mulheres inscritas em graduações nessa área diminuiu no decorrer dos anos [Nunes 2012]. Consequentemente, também ocorreu uma diminuição do número de mulheres presentes nos programas de pós-graduação e neste mercado de trabalho.

Nos últimos anos, o número de mulheres matriculadas em cursos da área de TI foi baixo quando comparado ao número de homens, que quase triplicou. Considerando os dados referentes ao número de egressos na área de computação, tem-se que em 2001 a porcentagem de concluintes mulheres era de $31.2 \%$, passou para $18.2 \%$ em $2010 \mathrm{e}$ diminuiu ligeiramente até 2012. Desta forma, percebe-se um baixo interesse feminino não só no ingresso, como também na conclusão dos cursos desta área [Nunes 2012].

Um exemplo desse cenário pode ser observado no curso de graduação em Sistemas de Informação da UFV - campus Rio Paranaíba. Conforme apresentado na Figura 1, no período de 2011 a 2014, observa-se que o percentual de mulheres entre todos os alunos que se matricularam no curso foi de 25\% em 2011, em 2012 caiu para 20\%, chegando a 13\% em 2013. Em 2014 observou-se um ligeiro aumento do número de mulheres, mas que não nos permite afirmar que há uma tendência de crescimento, visto que seria preciso analisar dados dos anos seguintes.

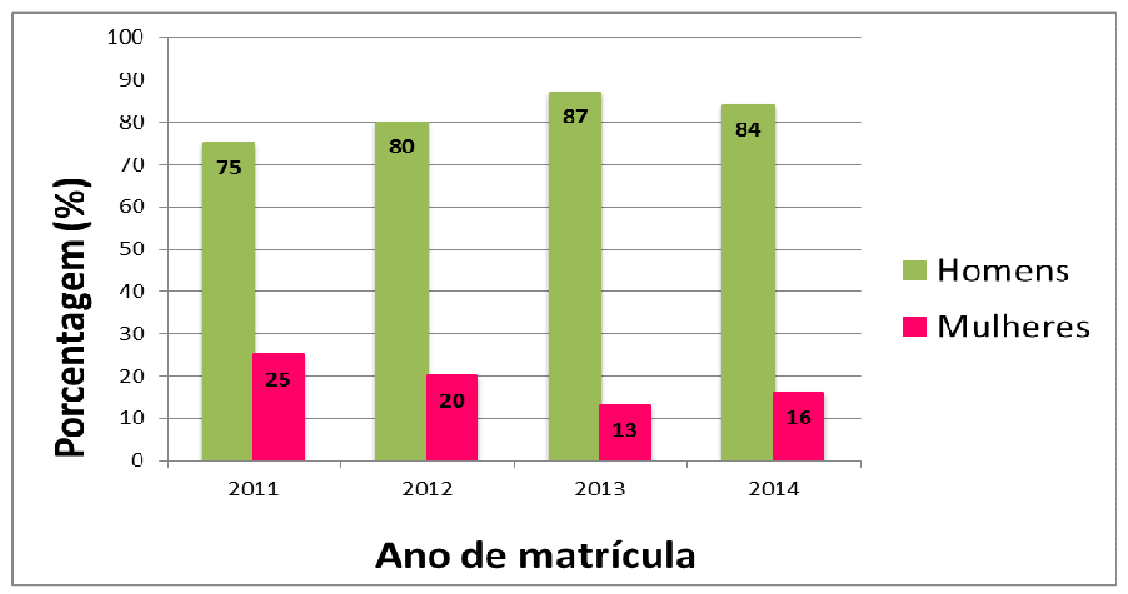

Figura 1 - Estudantes matriculados no curso de graduação em Sistemas de Informação da presente instituição

\footnotetext{
${ }^{1}$ http://timaior.mcti.gov.br/index.html
} 
As mulheres apresentam características importantes no âmbito profissional como: pontualidade, foco, e disposição para passar mais tempo no trabalho em que ingressam, visando o crescimento profissional. Elas têm investido mais em sua formação profissional buscando oportunidades além da graduação, como programas de Mestrado, Doutorado ou MBA [Gehringer 2012]. Elas possuem uma visão pontual associada a uma visão global para desempenhar suas tarefas, o que representa um diferencial no mercado de trabalho atual [The Drucker Institute 2006].

Considerando o potencial feminino e a falta de profissionais em TI observada atualmente, é desejável que cada vez mais mulheres sejam atraídas e absorvidas pelo mercado de trabalho nesta área. As causas para a minoria feminina têm sido investigadas e discutidas por diversas organizações [Ada Initiative 2015; Anita Borg Institute 2015; CSBC-WIT 2015] e trabalhos de pesquisa [Oliveira et al. 2010; Borges et al. 2010; Monard e Fortes 2013; Louzada et al. 2014]. Empresas importantes como IBM, Google, HP, Motorola, Facebook apoiam iniciativas que entendam melhor as causas do problema e promovam a atração de mulheres para a área de TI. A Google, por exemplo, possui um programa denominado "Google Women" que visa tratar da participação das mulheres na área de TI e já realizou o "Brazil Women in Technology Award", um prêmio voltado para inspirar mulheres estudantes da área de TI.

Neste contexto, este trabalho tem como objetivo o desenvolvimento de ações visando a atrair as estudantes do ensino médio para a área de TI. O trabalho foi desenvolvido por uma equipe formada por professoras e alunas pertencentes à graduação em Sistemas de Informação da UFV. As ações foram realizadas junto à única escola da cidade de Rio Paranaíba, voltada para o atendimento de alunos de $1^{\circ}, 2^{\circ}$ e $3^{\circ}$ ano do ensino médio.

Uma avaliação prévia realizada em 2013 com os alunos da escola mostrou que a maioria, incluindo as mulheres, possuía pouco conhecimento sobre as áreas de conhecimento, sobre os cursos de graduação oferecidos pela universidade federal presente no município e sobre a existência do curso de Sistemas de Informação [Silva e Reis 2015].

Este trabalho realizou diversas atividades ao longo do ano escolar de 2014 como palestras, dinâmicas e minicursos visando a promover a discussão sobre a importância da área de TI e da presença feminina em sua força de trabalho. As ações realizadas favorecem a complementação da formação dos estudantes e contribuem para seu futuro desenvolvimento profissional.

Este trabalho está estruturado da seguinte forma: a Seção 2 apresenta os trabalhos relacionados, a Seção 3 descreve a metodologia aplicada ao desenvolvimento do trabalho e a Seção 4 apresenta uma análise e discussão sobre os resultados alcançados com o trabalho. As conclusões são apresentadas na Seção 5.

\section{Trabalhos Relacionados}

Monard e Fortes (2013) realizaram uma pesquisa mais aprofundada sobre a participação de mulheres nos cursos de Ciência da Computação. Os resultados apresentados indicam que a presença feminina vem diminuindo no decorrer dos anos no Brasil. Louzada et al. (2014) também se concentraram neste tema, mapeando as publicações sobre o ingresso 
de mulheres na Computação e detalhando aspectos sobre a frequência anual de publicações sobre o tema e as abordagens usadas nas publicações.

No intuito de melhorar o cenário da minoria feminina na área de Tecnologia da Informação, diversas organizações nacionais e internacionais [Mulheres na Tecnologia 2015; Shepluplus 2015; Anita Borg Institute 2015] vêm trabalhando no sentido de reconhecer o potencial feminino na área. Outras iniciativas criadas por Instituições Federais de Ensino Superior têm sido apresentadas em eventos da Sociedade Brasileira de Computação [CSBC-WIT 2015, SBC 2015] para compartilhar experiências e desafios relacionados a trabalhos junto à comunidade, focadas em motivar estudantes do ensino médio a seguirem carreira na área de Computação.

Entre essas iniciativas, pode-se citar Gomes et al. (2014) que usaram a linguagem de programação Scratch com o objetivo de aumentar o interesse de meninas do ensino médio na área de computação. Foram alcançados resultados satisfatórios e foi possível estimular a capacidade de raciocínio das alunas que participaram do projeto, além de despertar mais uma possibilidade de carreira, pouco divulgada nas escolas.

Frigo et al. (2014) apresentaram um projeto com o objetivo de motivar meninas do ensino médio a seguirem a carreira nas áreas relacionadas as tecnologias computacionais. Para isso foram realizadas oficinas, palestras, vídeos e visitas. Os resultados apresentados foram satisfatórios e mostraram que as meninas que participaram do projeto gostaram do que aprenderam.

\section{Metodologia}

Este trabalho foi desenvolvido em duas etapas: (i) estudo sobre as causas do desinteresse feminino pela área de TI; (ii) desenvolvimento de atividades junto aos alunos de ensino médio na escola.

Inicialmente, foi realizada uma investigação sobre o desinteresse de mulheres na área de Computação. Para esta etapa, um questionário foi desenvolvido e aplicado aos alunos do ensino médio da escola e, paralelamente, foi realizada uma pesquisa na literatura. Na etapa seguinte, foi estabelecido um cronograma de atividades a serem desenvolvidas na escola. Cada atividade foi preparada e desenvolvida junto aos alunos dentro da escola e também, no laboratório de informática do curso de graduação em Sistemas de Informação.

O foco principal deste trabalho consistiu nas estudantes do sexo feminino. No entanto, as atividades foram realizadas envolvendo estudantes de ambos os gêneros. Essa conduta foi adotada para facilitar a aceitação deste trabalho por parte da escola e evitar qualquer tipo de segregação imprópria a um ambiente educacional. Desta forma, estudantes homens e mulheres participaram. Essas atividades complementam a formação dos alunos e contribuem para seu futuro desenvolvimento profissional. A escola forneceu seu apoio disponibilizando espaço físico e também, espaço em sua carga horária para a realização das atividades.

As atividades tiveram como público alvo estudantes do ensino médio, pois eles apresentam conhecimento insuficiente sobre os cursos de graduação existentes e estão mais próximos do momento de fazer a opção por uma profissão. Sobretudo, o trabalho 
priorizou os estudantes do $3^{\circ}$ ano do ensino médio, pois são aqueles que estão mais próximos de ingressar em uma graduação.

\section{Análise dos resultados}

Este trabalho foi realizado ao longo de todo o ano escolar de 2014. No primeiro contato, foram entrevistados 82 estudantes pertencentes às três turmas do $3^{\circ}$ ano do ensino médio, por meio de um questionário. Desses estudantes, $56(68,29 \%)$ eram do sexo feminino e apenas seis ( $11 \%$ das alunas) afirmaram ter interesse em cursar ensino superior na área de exatas, sendo que nenhuma pretendia optar por uma graduação na Computação/TI (Figura 2a).

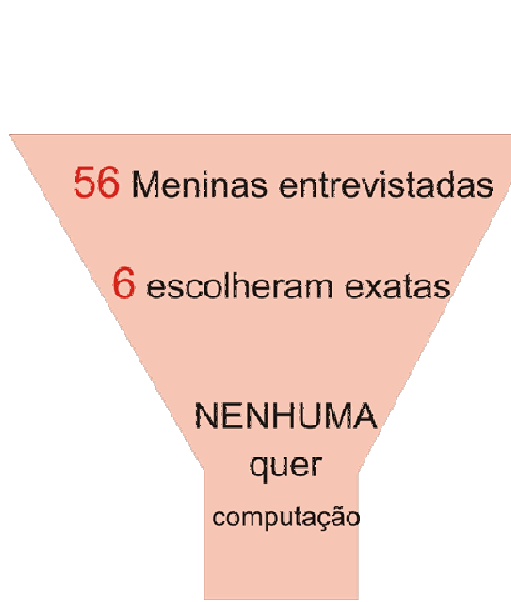

(a)

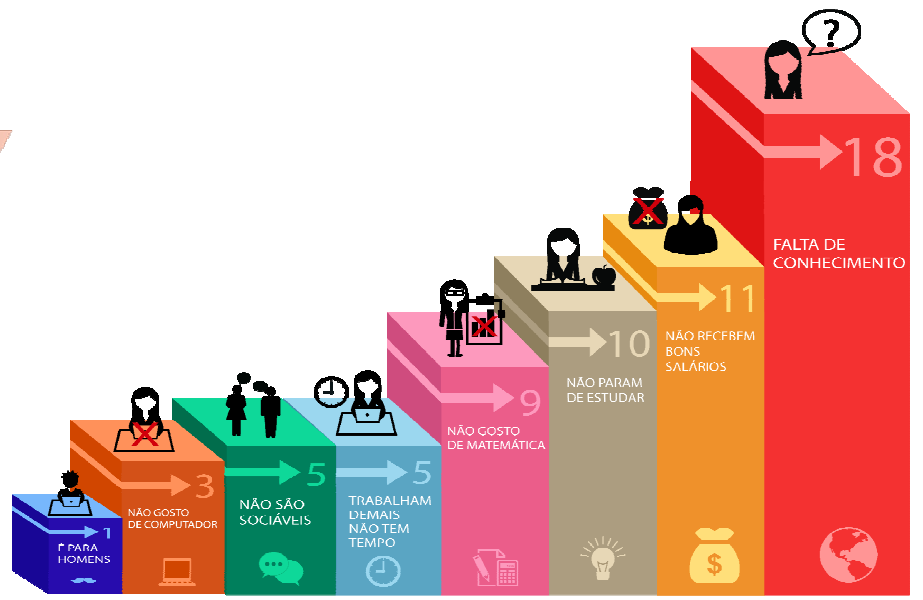

(b)

Figura 2. Avaliação do perfil dos estudantes antes do início das atividades

[fragmento extraído do questionário aplicado]
5 - Você já pensou na possibilidade de fazer um curso superior na área de
Computação?
( ) Sim
( ) Não
5.1 - Se você respondeu [Não] na questão anterior (questão 5), responda
quais motivos abaixo te levaram a não querer fazer o curso (marque uma ou
mais opções)
na área de Computação (marque quantas desejar)?
( ) Falta de conhecimento da área
( ) Não gosto de matemática
( ) Não gosto de computador ou tecnologia
( ) É um curso difícil, só alunos nerds podem fazer
( ) É um curso mais apropriado para homens
( ) É uma profissão que exige que a pessoa esteja sempre estudando mais
( ) Pessoas formadas em computação não são sociáveis
( ) Pessoas formadas em computação não recebem bons salários
( ) Pessoas formadas em computação trabalham demais e não tem tempo
para família e amigos.
( ) Outro —

Figura 3. Parte do questionário aplicado

Ainda sobre as respostas das alunas, quando questionadas se haviam pensado na possibilidade de optar por uma graduação na área de TI em algum momento, $77 \%$ das entrevistadas responderam que não. Os principais motivos apontados por elas para essa 
negativa (dentre os apresentados na Figura 3) foram: falta de conhecimento sobre a área (apontado por 18 alunas) e a impressão de que profissionais da área não recebem bons salários (apontado por 11 alunas) e precisam estudar muito (apontado por 10 alunas) (Figura 2b).

Durante todo o ano de 2014, foram realizados vários encontros na escola. Cada encontro teve a duração de 50 (cinquenta) minutos, que é a duração padrão da aula de qualquer disciplina. Um dos maiores desafios durante o trabalho foi desenvolver atividades que se adequassem a essa duração. A escola abriu espaço na carga horária dos alunos, pois a participação nas atividades mostrou-se mais efetiva quando as mesmas aconteciam durante o período regular de aula, do que em outro horário.

Em todos os encontros foram abordados temas relacionados à Tecnologia da Informação de forma dinâmica e criativa. As atividades foram desenvolvidas para abordar os temas através de uma linguagem mais adequada a esses alunos. Além das atividades, foram utilizados elementos cotidianos que fossem do interesse desses jovens como, por exemplo, jogos digitais e redes sociais on-line. As atividades foram realizadas separadamente com cada uma das turmas dos turnos da manhã e da tarde, pois foi observado que as atividades tinham sua efetividade comprometida se ultrapassassem certo número de participantes.

Os encontros realizados contemplaram palestras, dinâmicas, minicursos, visita à universidade e trabalho vocacional. As atividades foram estruturadas sempre visando à formação complementar dos alunos e seu futuro desenvolvimento profissional. Durante todas as atividades foi dada ênfase à importância da presença feminina na área de Computação. Para isso, o material utilizado favoreceu o papel da mulher em seus exemplos e ilustrações, além de mencionar os grandes nomes femininos da área. Dentre as atividades realizadas no encontro, algumas são descritas a seguir.

Palestra sobre as áreas de conhecimento: esta palestra apresentou aos alunos às áreas de conhecimento. Associado a esta palestra, os alunos participaram de uma dinâmica sobre números binários, que é um tema básico e fundamental para a área da computação (Figura 4a). Essa dinâmica foi realizada sem qualquer uso de computador e os conceitos dos números binários foram apresentados de forma lúdica, utilizando a técnica de Ciência da Computação Desplugada (Computer Science Unplugged) [Bell e Fellows, 2011]. Observou-se que os alunos aprenderam os conceitos e se surpreenderam. Eles relataram que o conteúdo era mais fácil do que eles julgavam anteriormente.

Palestra sobre profissões: esta palestra apresentou informações sobre cursos de graduação de todas as áreas de conhecimento, incluindo o curso de Sistemas de Informação. Foram priorizados os cursos de graduação presentes na UFV - campus Rio Paranaíba. Associado a esta palestra, os alunos participaram de uma dinâmica sobre programação em blocos utilizando um kit em papel (Figura 4b). A atividade permitiu mostrar aos alunos que a programação de computadores é mais simples do que pode parecer.

Mostra de profissões: a mostra proporcionou aos alunos informações sobre uma gama mais variada de profissões além daquelas apresentadas na palestra sobre profissões. Além disso, foram apresentadas as diferentes graduações existentes relacionados com a 
CBIE-LACLO 2015

Anais do XXI Workshop de Informática na Escola (WIE 2015)

área de Tecnologia da Informação (Ciência da Computação, Engenharia da Computação, entre outras).

Minicurso HTML e CSS: Os alunos participaram de minicurso sobre HyperText Markup Language - HTML e Cascading Style Sheets - CSS. O intuito do minicurso foi apresentar linguagens usadas para a produção de páginas Web e que fazem parte do contexto da área de Computação e de outras áreas (Figura 4c). Essas linguagens não envolvem um alto nível de complexidade e podem ser usadas por qualquer pessoa para criação de páginas Web.

Durante as atividades, a introdução de ensinamentos relativos aos fundamentos da computação e da programação enriqueceu a formação destes alunos, tornando-os mais aptos a atuar em uma sociedade onde ciência e inovação tem um papel fundamental.

A Figura 4(d) apresenta uma das turmas de $3^{\circ}$ ano que participaram das atividades. Além das atividades já descritas, também foi realizada visita dos alunos à universidade, palestra sobre conceitos de hardware e software e um curso de desenvolvimento de aplicativos para dispositivos móveis. Apenas esse último foi realizado exclusivamente com alunas mulheres.

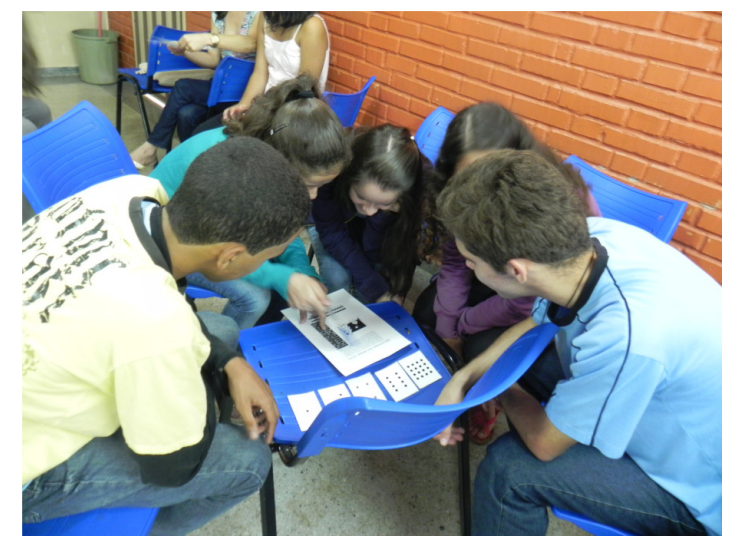

(a)

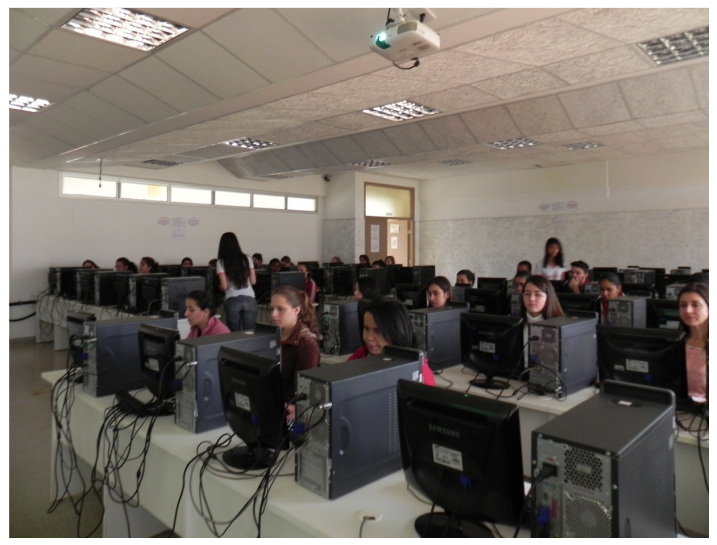

(c)

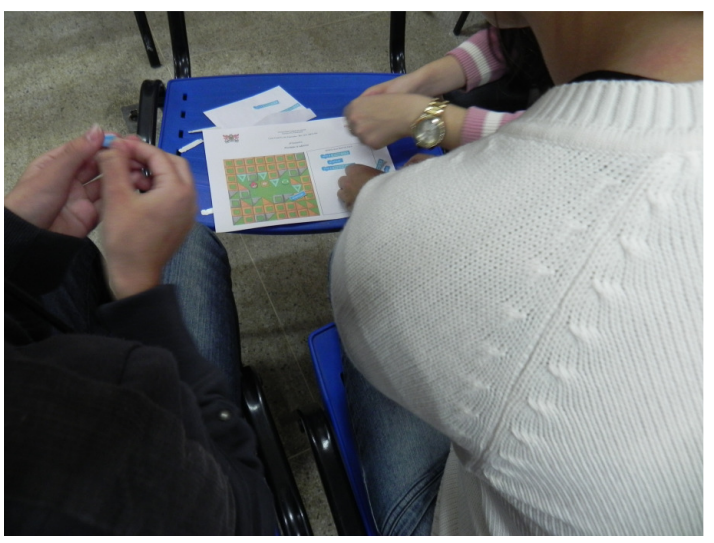

(b)

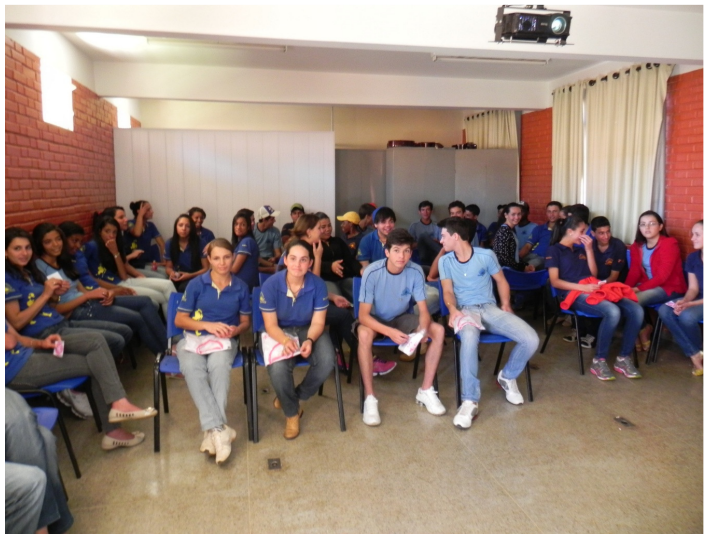

(d)

Figura 4 - Atividades realizadas com os alunos da escola

Ao longo da realização das atividades, foi possível observar pelo feedback dos alunos, que o trabalho realizado gerou impacto e transformações entre os estudantes, 
principalmente as meninas. Por meio da mídia social os estudantes foram estimulados a interagir e lá eles sinalizaram um retorno positivo sobre as atividades realizadas.

Foi realizada a aplicação de um novo questionário aos alunos do ensino médio da escola após a condução das atividades e antes da realização do Exame Nacional do Ensino Médio. O objetivo foi verificar eventuais mudanças ocorridas na escolha do curso superior por parte dos alunos. E investigar se essas mudanças tinham alguma relação com o efeito do trabalho realizado ao longo do ano.

Verificou-se que alunas do ensino médio mudaram de opinião em relação ao curso de graduação que pretendem cursar por influência das atividades realizadas por este trabalho. Em suas respostas, elas ressaltaram que as atividades foram um estímulo para se conhecer melhor os cursos de graduação. Segundo elas, as atividades também proporcionaram uma nova percepção em relação à área de Tecnologia da Informação. Além disso, $79 \%$ das alunas e $88 \%$ dos alunos que participaram das atividades indicaram que gostariam de participar de novos projetos focados na área de Computação (Figura 5). Esses dados sinalizam que o trabalho promoveu uma consciência sobre a importância da área por parte da maioria geral dos alunos. De forma geral, os estudantes declararam que as atividades mais atraentes foram aquelas que envolveram visitas e atividades práticas no laboratório de informática da nossa universidade, permitindo conhecer o desenvolvimento de programas de computador e suas respectivas linguagens.

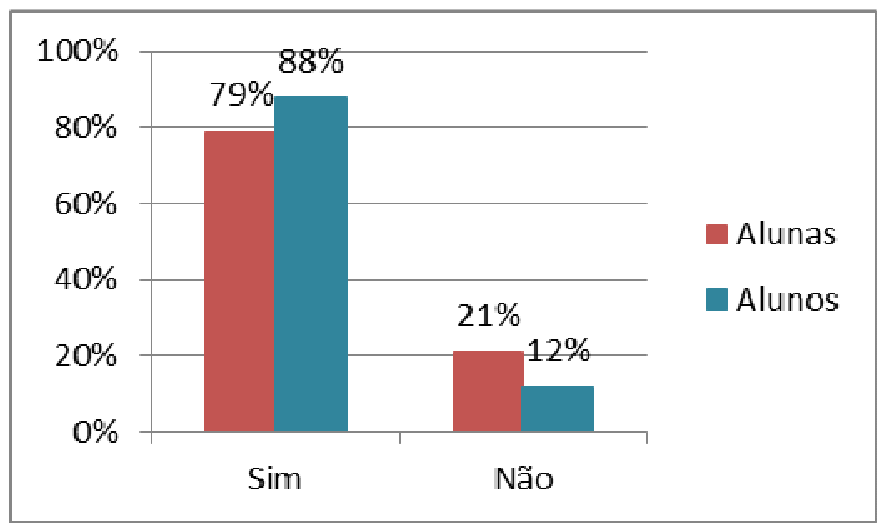

Figura 5 - Percentual de alunas e alunos que gostariam de participar de novos projetos focados na área de Computação

A escola em questão é a única do município a oferecer ensino médio e desta forma absorve os alunos da cidade e do meio rural. Esses alunos e também os professores da escola passaram a ter um conhecimento que pode ser aplicado no seu dia a dia profissional e pessoal. Em particular, considerando os alunos provenientes do meio rural, as informações apresentadas por este trabalho sobre a área de Computação, suas possibilidades de mercado e a atuação feminina nesse setor; atingiram um público que em geral tem mais contato com informações sobre outras áreas ligadas a Ciências Biológicas e Agrárias.

\section{Conclusão}

Ao longo do desenvolvimento do trabalho, foram realizados diversos encontros incluindo palestras, minicursos e dinâmicas. As dinâmicas promovidas foram voltadas para a área de Computação com o uso de metodologias lúdicas (e.g. técnicas de 
computação desplugada) para explicar conceitos da área. Todo o material usado deu ênfase à importância da presença feminina na área de Computação por meio de exemplos, ilustrações e menção aos grandes nomes femininos da área. A abordagem empregada contribuiu para favorecer a aceitação deste trabalho por parte da escola.

Até o momento foi possível observar que trabalho tem gerado impactos e transformações. Quando o trabalho teve início, nenhuma das alunas sinalizava intenção de fazer um curso superior na área de Computação em função do desconhecimento sobre a área. Entretanto, nos últimos encontros, observou-se que algumas alunas sinalizavam interesse em saber mais da área de TI e revelaram ter mudado um pouco a visão que tinham sobre área.

Além disso, as atividades promoveram junto à maioria dos alunos, um melhor entendimento sobre a área de TI e sua importância na sociedade atual pautada cada vez mais no desenvolvimento da ciência, tecnologia e inovação. A maioria dos estudantes (meninos e meninas) sinalizaram que gostariam de participar de novos projetos focados na área de computação.

Isso mostra que este trabalho está cumprindo sua missão. Espera-se continuar a realizar ações para despertar o interesse do maior número possível de talentos do sexo feminino para a área de TI. Esse mercado ainda conta com um número insuficiente de profissionais qualificados e o número de mulheres é muito menor do que seria o ideal. Além disso, o trabalho promove indiretamente a formação complementar de estudantes do sexo masculino, pois a maioria das atividades inclui alunos de ambos os gêneros.

O trabalho desenvolvido na escola em 2014 apresentou um alcance local, mas se mostrou bastante efetivo. Nossa pretensão é ampliar o escopo de atuação do trabalho e aplicar as atividades a outras escolas da região. E posteriormente, em outras cidades mais distantes.

\section{Agradecimentos}

Ao MCTI/CNPq/SPM-PR/Petrobras que fomentaram o desenvolvimento deste trabalho.

\section{Referências}

Ada Initiative (2011) "Who we are". Disponível em: <https://adainitiative.org/>. Acesso em: 10/05/2015.

Anita Borg Institute (2015). "Anita Borg Institute for Women and Tecnology". Disponível em: <http://anitaborg.org/>. Acesso em: 10/03/2015.

Bell, T., Witten, I. H., Fellows, M. (2011). “Computer Science Unplugged: Ensinando Ciência da Computação sem uso do computador". Tradução por Luciano Porto Barreto. Disponível em: <http://csunplugged.org>.

Borges, K. F. C., Ide, M. H. S., Durães, S. J. A. (2010) "Mulheres na educação superior no Brasil: estudo de caso do Curso de Sistema de Informação da Universidade Estadual de Montes Claros (2003/2008)", VIII Congresso Iberoamericano de Ciência, Tecnologia e Gênero.

CSBC-WIT (2015). "WIT - Women in Information Technology". Disponível em: $<$ http://csbc2015.cin.ufpe.br/eventos_descricao/9 />. Acesso em 10/03/2015. 
Frigo, L., Cardoso, P., Cardoso, J. P., Coelho, M. H., Pozzebon, E. (2014) "Prática de Incentivo à Inserção de Mulheres nos Cursos Tecnológicos e de Engenharia: Robótica Educacional". In: XL Conferência Latinoamericana en Informática, 2014, Montevidéu. Memorias del VI Congreso de la Mujer Latinoamericana en la Computación (LAWCC 2014), v. 1. p. 31-35.

Gehringer, M. (2012) "Mulheres levam vantagem nas seleções de estagiários". Podcast disponível em: <http://cbn.globoradio.globo.com/comentaristas/maxgehringer/2012/10/09/MULHERES-LEVAM-VANTAGEM-NAS-SELECOES-DEESTAGIARIOS.htm>. Acesso em: 13/05/2015.

Gomes, W. F., Louzada, C. S., Nunes, M. A. S. N., Salgueiro, E. M., Trinchão, B. (2014) "Incentivando meninas do ensino médio à área de Ciência da Computação usando o Strach como ferramenta", 20ª Workshop de Informática na Escola (WIE).

Louzada, C. S., Gomes, W.F., Nunes, M. A. S. N., Salgueiro, E. M., Trinchão, B. (2014) "Um Mapeamento das publicações sobre o ingresso das mulheres na computação", VI Congresso de la Mujer Latinoamericana em La Computacion. LAWCC 2014.

Monard, M. C., Fortes, R. P. M. (2013). "Uma Visão da Participação Feminina nos Cursos de Ciência da Computação no Brasil", V Congresso de la Mujer Latinoamericana em La Computacion. LAWCC 2013.

Mulheres na Tecnologia (2015) "Mulheres na Tecnologia". Disponível em: $<$ http://mulheresnatecnologia.org >. Acesso em: 10/03/2015.

Nunes, D. J. (2012) “Educação Superior em Computação, Estatísticas 2012”. Sociedade Brasileira de Computação. Disponível em: $<$ http://www.sbc.org.br/index.php?option=com_jdownloads\&Itemid=195\&task=vie w.download\&catid=39\&cid=567>. Acesso em: 13/05/2015.

Oliveira, D. V., Ambrósio, A. P., Alvares, N. O. (2010) "As mulheres no curso de ciências da computação da Universidade Federal de Goiás". In: LAWCC 2010 Latin American Women in Computer Science - San Lorenzo - Paraguay.

SBC (2015). Sociedade Brasileira de Computação - Notícias, 2013. Disponível em: $<\mathrm{http}: / / \mathrm{www}$. sbc.org.br/index.php?option=com_content\&view=article\&id=865:proje to-meninas-digitais-discussoes-workshops-e-motivacao\&catid=65:destaquesp $>$. Acesso em: 10/03/2015.

Shepluplus (2015) "She++". Disponível em: <http://sheplusplus.stanford.edu/ >. Acesso em: 10/03/2015.

Silva, G. R., Reis, R. C. D. (2015). Meninas++ - A brazilian project to attract more girls to IT. New York Celebration of Women in Computing (NYCWiC), Syracuse, NY.

The Drucker Institute (2006) "Drucker's Life and Legacy". Disponível em: < http://www.druckerinstitute.com/link/about-peter-drucker/>. Acesso em 10/03/2015.

Venceslau, M. (2013) "Falta de profissionais de TI se agravará no Brasil, diz IDC". Disponível em: <http://exame.abril.com.br/carreira/noticias/falta-de-profissionais-deti-se-agravara-no-brasil-diz-idc>. Acesso em: 14/05/2015 